\title{
SOME INEQUALITIES FOR NORM UNITARIES IN BANACH ALGEBRAS
}

\author{
by M. J. CRABB and J. DUNCAN
}

(Received 11th June 1976)

\section{Introduction}

Let $A$ be a complex unital Banach algebra. An element $u \in A$ is a norm unitary if

$$
\|u\|=\left\|u^{-1}\right\|=1 \text {. }
$$

(For the algebra of all bounded operators on a Banach space, the norm unitaries are the invertible isometries.) Given a norm unitary $u \in A$, we have $\operatorname{Sp}(u) \subset \Gamma$, where $\operatorname{Sp}(u)$ denotes the spectrum of $u$ and $\Gamma$ denotes the unit circle in $C$. If $\operatorname{Sp}(u) \neq \Gamma$ we may suppose, by replacing $u$ by $e^{i \theta} u$, that $-1 \notin \mathrm{Sp}(u)$. Then there exists $h \in A$ such that

$$
\left.u=e^{i h}, \quad \operatorname{Sp}(h) \subset\right]-\pi, \quad \pi[.
$$

Since $\left\|u^{n}\right\|=1 \quad(n \in Z)$ it follows that $\left\{e^{i \xi h}: \xi \in R\right\}$ is norm bounded; thus $h$ is Hermitian equivalent (see $(4,85)$ ), i.e. there exists an equivalent algebra norm $|$.$| on A$ for which $\left|e^{i \xi h}\right|=1(\xi \in R)$. Prompted by questions of L. A. Harris concerning $J^{*}$-algebras and Banach Lie groups (see (5)), we wish to determine the best bounds for $\left\|e^{i \xi h}\right\|$ without renorming. To be precise we wish to determine the best bound for $\left\|e^{i \xi h}\right\|$ subject to $\left\|e^{ \pm i h}\right\|=1, r(h) \leqslant \tau$ where $r(h)$ denotes the spectral radius of $h$ and $0<\tau<\pi$. By using results on entire functions of exponential type we are able to give precise best bounds for some cases in which $\tau$ is a rational multiple of $\pi$. We show that the extremal algebra subject to $\left\|e^{ \pm i h}\right\|=1, r(h) \leqslant \tau$ can be identified with a quotient algebra of the Wiener algebra. By the same technique we are able to refine $S$. Kaijser's generalisation of a result of $\mathrm{N}$. Th. Varopoulos (6) concerning inequalities between $r\left(e^{i h}-1\right)$ and $\left\|e^{i h}-1\right\|$.

\section{Preliminaries on entire function of exponential type}

Let $g$ be an entire function and let

$$
M(r)=\sup \{|g(z)|:|z|=r\} .
$$

We recall that $g$ is of $\operatorname{order} \rho$ if

$$
\limsup _{r \rightarrow \infty} \frac{\log \log M(r)}{\log r}=\rho
$$

and then $g$ is of type $\tau$ if

$$
\limsup _{r \rightarrow \infty} r^{-\rho} \log M(r)=\tau
$$


An entire function $g$ is of exponential type $\tau$ if $g$ has order less than 1 or if $g$ has order 1 and type equal to or less than $\tau$.

Let $\mathscr{F}(\tau)$ be the set of all entire functions $g$ of exponential type $\tau$ for which

$$
|g(k)| \leqslant 1 \quad(k \in Z)
$$

and let

$$
\mu(\tau)=\sup \{|g(\xi)|: \xi \in R, \quad g \in \mathscr{F}(\tau)\}
$$

We shall use the following results, most of which are implicit in R. P. Boas (2).

Theorem 1. Let $g \in \mathscr{F}(\tau)$ where $0<\tau<\pi$.

(i) For $\xi \in \boldsymbol{R} \backslash \boldsymbol{Z}$,

$$
g(\xi)=\frac{\sin \pi \xi}{\pi(\pi-\tau)} \sum_{k \in Z} \frac{(-1)^{k} g(k)}{(k-\xi)^{2}} \sin [(\pi-\tau)(\xi-k)] .
$$

(ii) For $0<\alpha<1, \beta=1-\alpha$,

$$
g(0) \sin \beta \tau+g(1) \sin \alpha \tau=\sum_{k \in Z} c_{k} g(\alpha+k \pi / \tau)
$$

where

$$
c_{k}=(-1)^{k} \frac{\sin \alpha \tau \sin \beta \tau}{(\alpha+k \pi / \tau)(\beta-k \pi / \tau)}
$$

(iii) $\mu(\tau) \leqslant \sec \frac{1}{2} \tau$.

(iv) $|g(1)-g(0)| \leqslant 2 \sin \frac{1}{2} \tau \sup \left\{\left|g\left(\frac{1}{2}+\left(k-\frac{1}{2}\right) \pi / \tau\right)\right|: k \in Z\right\}$.

Proof. (i) In $(2,(10.2 .9))$ let $\delta \rightarrow \pi-\tau$ and apply the dominated convergence theorem.

(ii) Apply the method of $(2, \S 11.2)$. In the notation of Boas, take

$$
\lambda(\xi)=e^{-i \alpha \xi} \sin \beta \tau+e^{i \beta \xi} \sin \alpha \tau .
$$

Note that $\lambda(\tau)=\lambda(-\tau)$ and $\lambda(\xi)=\Sigma_{k \in Z} c_{k} e^{i k \pi \xi \tau}$ with $c_{k}$ as in the statement of the theorem. Since

$$
L[g(z)]=g(z-\alpha) \sin \beta \tau+g(z+\beta) \sin \alpha \tau
$$

we conclude that

$$
g(\xi-\alpha) \sin \beta \tau+g(\xi+\beta) \sin \alpha \tau=\sum_{k \in Z} c_{k} g(\xi+k \pi / \tau) .
$$

Put $\xi=\alpha$.

(iii) Given $g \in \mathscr{F}(\tau), k \in Z$, we note that $z \rightarrow g(z+k)$ is also in $\mathscr{F}(\tau)$. It follows that

$$
\mu(\tau)=\sup \{|g(\xi)|: 0 \leqslant \xi \leqslant 1, g \in \mathscr{F}(\tau)\} .
$$

By Montel's theorem and the compactness of $[0,1]$, there exists $g_{0} \in \mathscr{F}(\tau)$ and $0<\alpha<1$ such that $\mu(\tau)=\left|g_{0}(\alpha)\right|$. By applying part (ii) with $g(z)=\cos \tau\left(z-\frac{1}{2}\right)$ we find that 


$$
\cos \frac{1}{2} \tau(\alpha-\beta)\left[\left|c_{0}\right|-\sum_{k \neq 0}\left|c_{k}\right|\right]=\cos \frac{1}{2} \tau[\sin \alpha \tau+\sin \beta \tau] .
$$

By applying part (ii) with $g_{0}$ we find that

$$
\begin{aligned}
\sin \alpha \tau+\sin \beta \tau & \geqslant\left|g_{0}(1) \sin \alpha \tau+g_{0}(0) \sin \beta \tau\right| \\
& =\left|\sum_{k \in Z} c_{k} g_{0}(\alpha+k \pi / \tau)\right| \\
& \geqslant\left|c_{0}\right|\left|g_{0}(\alpha)\right|-\mu(\tau) \sum_{k \neq 0}\left|c_{k}\right| \\
& =\mu(\tau)\left[\left|c_{0}\right|-\sum_{k \neq 0}\left|c_{k}\right|\right] .
\end{aligned}
$$

It follows that

$$
\mu(\tau) \leqslant \sec \frac{1}{2} \tau \cos \tau\left(\alpha-\frac{1}{2}\right) \leqslant \sec \frac{1}{2} \tau .
$$

(iv) This follows from the details of the proof of $(2,(11.4 .2))$.

For a discussion of estimates for $\mu(\tau)$, see (2, p. 203). In particular it should be noted that there is some overlap between the results in this paper and the work of $S$. $\mathrm{N}$. Bernstein (1), although rather different arguments are employed by Bernstein. The exact value of $\mu(\tau)$ is given below for the cases $\tau=\pi / m, \pi(1-1 / m)$ where $m \in N$, $m \geqslant 2$.

\section{Estimates for $\left\|e^{i \xi h}\right\|$}

Theorem 2. Let $h \in A$ with $\left\|e^{ \pm i h}\right\|=1$ and $r(h) \leqslant \tau$.

(i) If $\tau=\pi / m$ with $m \in N, m \geqslant 2$, then $\left\|e^{i \xi h}\right\| \leqslant \phi_{m}(\xi)(0 \leqslant \xi \leqslant 1)$ where

$$
\phi_{m}(\xi)=\sec \frac{1}{2} \pi / m \cos \left(\xi-\frac{1}{2}\right) \pi / m \text {. }
$$

(ii) If $\tau=\pi(1-1 / m)$ with $m \in N, m \geqslant 2$, then $\left\|e^{i \xi h}\right\| \leqslant \psi_{m}(\xi)(0 \leqslant \xi \leqslant 1)$ where

$$
\psi_{m}(\xi)=\frac{\sin \pi \xi}{m} \sum_{k=1}^{m} \operatorname{cosec}(k-\xi) \pi / m \text {. }
$$

The above inequalities are best possible.

Proof. Given $f \in A^{\prime},\|f\| \leqslant 1$, let $g(z)=f\left(e^{i z h}\right)(z \in C)$. Then $g$ is entire and $|g(z)| \leqslant\left\|e^{i z h}\right\|$. Since $r(h) \leqslant \tau$ it follows that $g$ is of exponential type $\tau$, and since $\left\|e^{i n h}\right\|=1(n \in Z)$ we have $g \in \mathscr{F}(\tau)$. To obtain estimates for $\left\|e^{i \xi h}\right\|$ it is now sufficient, by the Hahn-Banach theorem, to obtain the corresponding estimates for $|g(\xi)|$ with $g \in \mathscr{F}(\tau)$.

(i) Let $\tau=\pi / m, m \in N, m \geqslant 2$. Given $0<\xi<1$ let

$$
\mu_{\xi}=\sup \{|g(\xi)|: g \in \mathscr{F}(\tau)\} .
$$

As in the proof of Theorem 1 (iii) there exists $g_{0} \in \mathscr{F}(\tau)$ with $\left|g_{0}(\xi)\right|=\mu_{\xi}$ and the same 
argument gives

$$
\mu_{\xi} \leqslant \sec \frac{1}{2} \tau \cos \tau\left(\xi-\frac{1}{2}\right) .
$$

(ii) Let $\tau=\pi(1-1 / m), m \in N, m \geqslant 2$. Note that $\psi_{m}$ is of exponential type $\tau$. For $k=1,2, \ldots, m$ and $0 \leqslant \xi \leqslant 1$, we have $\psi_{m}(k)=(-1)^{k-1}, \sin (\pi-\tau)(\xi-k)<0$, and hence

$$
(-1)^{k} \psi_{m}(k) \sin (\xi-k) \pi / m=|\sin (\xi-k) \pi / m| .
$$

This last equation holds for all $k$ since each side is unchanged when $k$ is replaced by $k+m$. Given $g \in \mathscr{F}(\tau)$, Theorem 1 (i) now gives for $0<\xi<1$,

$$
\begin{aligned}
|g(\xi)| & \leqslant \frac{m \sin \pi \xi}{\pi^{2}} \sum_{k \in Z} \frac{|\sin (\xi-k) \pi / m|}{(k-\xi)^{2}} \\
& =\frac{m \sin \pi \xi}{\pi^{2}} \sum_{k \in Z} \frac{(-1)^{k} \psi_{m}(k)}{(k-\xi)^{2}} \sin (\xi-k) \pi / m \\
& =\psi_{m}(\xi) .
\end{aligned}
$$

Finally we show that these inequalities are best possible, even for finite dimensional algebras. We write

$$
\omega_{s}(t)=e^{i s t} \quad(t \in R) .
$$

For the case $\tau=\pi / m$, let $E_{m}$ be the linear span of $\omega_{\pi / m}, \omega_{-\pi / m}$ with norm defined by

$$
\|g\|=\sup \{|g(k)|: k \in Z\} .
$$

(Theorem 1(i) shows that $g=0$ whenever $\|g\|=0$.) Let $D$ be the bounded linear operator on $E_{m}$ given by

$$
D g=-i g^{\prime} \quad\left(g \in E_{m}\right) .
$$

Then $\operatorname{Sp}(D)=\{ \pm \pi / m\}$. Taylor's theorem gives

$$
e^{i \xi D} g(t)=g(\xi+t)
$$

and hence $\left\|e^{ \pm i D}\right\|=1$. Consideration of the function

$$
g(t)=\cos \left(t-\frac{1}{2}\right) \pi / m
$$

shows that $\left\|e^{i \xi D}\right\| \geqslant \phi_{m}(\xi)(0 \leqslant \xi \leqslant 1)$, as required. For the case $\tau=\pi(1-1 / m)$ let $F_{m}$ be the linear span of

$$
\left\{\omega_{k \pi / m}: k=0, \pm 1, \pm 2, \ldots, \pm(m-1)\right\} .
$$

With $\|$.$\| and D$ as above we obtain $\left\|e^{ \pm i D}\right\|=1, r(D)=\pi(1-1 / m)$. Since $\psi_{m} \in F_{m}$ we also obtain $\left\|e^{i \xi D}\right\| \geqslant \psi_{m}(\xi)(0 \leqslant \xi \leqslant 1)$, as required.

Let $W$ denote the Wiener algebra of all continuous functions $x$ on $[-\pi, \pi]$ with absolutely convergent Fourier series

$$
x(t)=\sum_{k \in Z} c_{k} e^{i k t} \quad(-\pi \leqslant t \leqslant \pi)
$$

and with the norm 


$$
\|x\|=\sum_{k \in \mathbf{Z}}\left|c_{k}\right|
$$

Given $0<\tau<\pi$ let $\operatorname{ker}[-\tau, \tau]$ be the closed ideal of $W$ given by

$$
\operatorname{ker}[-\tau, \tau]=\{x \in W: x(t)=0 \quad(-\tau \leqslant t \leqslant \tau)\} .
$$

Let $\left(A_{\tau},\|\cdot\|_{\tau}\right)$ denote the quotient algebra $W / \operatorname{ker}[-\tau, \tau]$. It is elementary that $A_{\tau}$ may be identified with the algebra of continuous functions $x$ on $[-\tau, \tau]$ which have representations

$$
x(t)=\sum_{k \in Z} c_{k} e^{i k t} \quad(-\tau \leqslant t \leqslant \tau)
$$

and then

$$
\|x\|_{r}=\inf \left\{\sum_{k \in Z}\left|c_{k}\right|: \text { all such representations }\right\} .
$$

We note that $\eta \in A_{\tau}$ where $\eta(t)=t(-\tau \leqslant t \leqslant \tau)$. It is now a standard argument to show that $A_{\tau}$ is the extremal algebra with respect to the constraints $\left\|e^{ \pm i h}\right\|=1, r(h) \leqslant \tau$. In particular we have the following result.

Theorem 3. Let $h \in A$ with $\left\|e^{ \pm i h}\right\|=1, r(h) \leqslant \tau$, where $0<\tau<\pi$. Then for $0 \leqslant \xi \leqslant 1$

$$
\left\|e^{i \xi \hbar}\right\| \leqslant\left\|e^{i \xi \eta}\right\|_{r}=\sup \{|g(\xi)|: g \in \mathscr{F}(\tau)\}
$$

Proof. Let $\left\{c_{k}\right\}$ be any absolutely summable sequence with

$$
e^{i \xi t}=\sum_{k \in Z} c_{k} e^{i k t} \quad(-\tau \leqslant t \leqslant \tau)
$$

For any entire function $g$ of exponential type $\tau$ bounded on $\boldsymbol{R}$ the method of $(2, \S 11.2)$ gives

$$
g(\xi)=\sum_{k \in Z} c_{k} g(k)
$$

In particular, given $f \in A^{\prime}$ let

$$
g(z)=f\left(e^{i z h}\right) \quad(z \in C)
$$

and we obtain

$$
f\left(e^{i \xi h}\right)=\sum_{k \in Z} c_{k} f\left(e^{i k h}\right)=f\left(\sum_{k \in Z} c_{k} e^{i k h}\right) .
$$

The Hahn-Banach theorem gives

$$
e^{i \xi h}=\sum_{k \in Z} c_{k} e^{i k h}
$$

and so

$$
\left\|e^{i \xi h}\right\| \leqslant \sum_{k \in Z}\left|c_{k}\right| .
$$

Since this holds for all representations of $e^{i \xi t}$ we conclude that $\left\|e^{i \xi h}\right\| \leqslant\left\|e^{i \xi \eta}\right\|_{r}$. 
The method of (3) shows that $A_{r}^{\prime}$ may be identified with the space of all entire functions $g$ of exponential type $\tau$ bounded on $R$ with norm given by

$$
\|g\|=\sup \{|g(k)|: k \in Z\} .
$$

Under the identification we obtain

$$
\left\|e^{i \xi \eta}\right\|_{\tau}=\sup \{|g(\xi)|: g \in \mathscr{F}(\tau)\}
$$

Remarks. (1) We note that for $m \in N, m \geqslant 2$

$$
\begin{aligned}
& \mu(\pi / m)=\sec \left(\frac{1}{2} \pi / m\right)=1+O\left(m^{-2}\right) \\
& \mu(\pi(1-1 / m))=\frac{1}{m} \sum_{k=1}^{m} \operatorname{cosec}\left(k-\frac{1}{2}\right) \pi / m=O(\log m) .
\end{aligned}
$$

(2) Given $\left\|e^{ \pm i h}\right\|=1, r(h) \leqslant \tau$, we need not have $h$ Hermitian no matter how small $\tau$ is. By taking a direct sum of the spaces $E_{m}$ of Theorem 2 we may obtain a Banach space $X$ for which there is no $\epsilon>0$ such that every invertible isometry $L$ with $\|I-L\|<\epsilon$ is of the form $L=e^{i H}$ with $H$ Hermitian. This is essentially the example in (5) in which the group of invertible isometries of $X$ is not a Banach Lie group.

(3) Given $\left\|e^{ \pm i h}\right\|=1, r(h)<\pi$ there is no uniform bound on $\left\|e^{(1 / 2) i h}\right\|$.

(4) When $\tau=\frac{1}{2} \pi$ it is easily verified that the operator $D$ on $E_{2}$ is isometrically equivalent to the matrix

$$
\left(\begin{array}{cc}
0 & \frac{1}{2} \pi \\
\frac{1}{2} \pi & 0
\end{array}\right)
$$

acting on $C^{2}$ with the sup norm.

(5) It would be useful to have a periodic extension of $e^{(1 / 2) i t}$ from $(-\tau, \tau]$ to $[-\pi, \pi]$ whose Fourier coefficients give $\left\|e^{(1 / 2) i \eta}\right\|_{\tau}$. For the case $\tau=\pi(1-1 / m)$ it may be verified that the required extension is provided by

$$
\frac{m}{\pi}(\pi-t) e^{(1 / 2) i t} \quad(\pi-\pi / m \leqslant t \leqslant \pi+\pi / m) .
$$

For the case $\tau=\pi / m$ the extension is again of the form $\chi(t) e^{(1 / 2) i t}$ where $\chi$ is piecewise linear. It would be of interest to resolve the case $\tau=r \pi$ where $r$ is rational, $0<r<1$.

\section{Estimates for $\left\|e^{i h}-1\right\|$}

In the course of proving that Kronecker sets are Helson sets, Varopoulos (6) established for the Wiener algebra that if $\left\|u_{n}\right\|=\left\|u_{n}^{-1}\right\|=1$ and $r\left(u_{n}-1\right) \rightarrow 0$ then $\left\|u_{n}-1\right\| \rightarrow 0$. S. Kaijser informed us that the argument in (6) would give for an arbitrary Banach algebra essentially the following result.

"If $\|u\|=\left\|u^{-1}\right\|=1, r(u-1) \leqslant 2 \sin \pi / 4 m$ where $m \in N$, then $\|u-1\| \leqslant \pi / m e^{(1 / 2) \pi / m}$.",

We give below a sharper version of this (unpublished) result of Kaijser.

Theorem 4. Let $\|u\|=\left\|u^{-1}\right\|=1, r(u-1) \leqslant 2 \sin \frac{1}{2} \tau$ where $0<\tau<\pi$.

(i) $\|u-1\| \leqslant 2 \tan \frac{1}{2} \tau$. 
(ii) The inequality is best possible if $\tau=\pi / m, m \in N, m$ even.

(iii) If $\tau=\pi / m, m \in N, m$ odd, then $\|u-1\| \leqslant 2 \sin \frac{1}{2} \tau$. let

Proof. (i) The conditions on $u$ give $u=e^{i h}, r(h) \leqslant \tau$. Let $f \in A^{\prime}$ with $\|f\| \leqslant 1$ and

$$
g(z)=f\left(e^{i z h}\right) \quad(z \in C) .
$$

Theorem 1 (iii) and (iv) gives

$$
|g(1)-g(0)| \leqslant 2 \sin \frac{1}{2} \tau \sec \frac{1}{2} \tau=2 \tan \frac{1}{2} \tau
$$

and the Hahn-Banach theorem gives $\|u-1\| \leqslant 2 \tan \frac{1}{2} \tau$.

(ii) Let $\tau=\pi / m, m \in N, m$ even. Let $E_{m}, D$ be as in Theorem 2 . Then

$$
\left(e^{i D}-I\right) g(0)=g(1)-g(0)
$$

and the function $g(t)=\sec \frac{1}{2} \pi / m \sin \left(t-\frac{1}{2}\right) \pi / m$ gives

$$
\left\|e^{i D}-I\right\| \geqslant 2 \tan \frac{1}{2} \pi / m \text {. }
$$

(iii) For the case $m$ odd, Theorem 1(iv) gives

$$
|g(1)-g(0)| \leqslant 2 \sin \frac{1}{2} \tau \sup \{|g(k)|: k \in Z\} \leqslant 2 \sin \frac{1}{2} \tau
$$

and hence $\|u-1\| \leqslant 2 \sin \frac{1}{2} \tau$.

Corollary. If $\|u\|=\left\|u^{-1}\right\|=1, r(u-1)=2 \sin \frac{1}{2} \pi / m$ where $m \in N, m$ odd, then $\|u-1\|=r(u-1)$.

\section{REFERENCES}

(1) S. N. BERNSTEIN, The extension of properties of trigonometric polynomials to entire functions of finite degree, Izv. Akad. Nauk, 12 (1948), $421-444$ (Russian).

(2) R. P. BOAS, Entire functions (Academic Press, 1954).

(3) B. Bollobís, The numerical range in Banach algebras and complex functions of exponential type, Bull. London Math. Soc. 3 (1971), 27-33.

(4) F. F. BONSALL and J. DUNCAN, Numerical ranges of operators on normed spaces and of elements of normed algebras (London Math. Soc. Lecture Note Series, No. 2, Cambridge University Press, 1971).

(5) L. A. HARRIS and W. KAUP, Linear algebraic groups in infinite dimensions, Illinois $J$. Math. 21 (1977), 666-674.

(6) N. Th. VAROPOulos, Sur les ensembles parfaits et les series trigonométriques, C.R. Acad. Sci. Paris 260 (1965), 3831-3834. 